\title{
Interventions to reduce urinary catheter use: it worked for them, but will it work for us?
}

\section{Jennifer Meddings}

\section{Correspondence to}

Dr Jennifer Meddings, Department of Internal Medicine, University of Michigan Medical School, 2800 Plymouth Road, Building 16, 430W, Ann Arbor, MI 48109, USA; meddings@umich.edu

Accepted 18 July 2013 Published Online First 19 September 2013

\section{SLinked}

http://dx.doi.org/10.1136/ bmjqs-2013-001908

To cite: Meddings J. BMJ Qual Saf 2013;22:967-971.
In most hospitals, four steps are required to remove a urinary catheter ${ }^{1}$ : the physician recognises the patient has a catheter in place; the physician realises the catheter is no longer necessary; the order is written to remove the catheter; and the nurse removes the catheter. Interventions to prompt removal of unnecessary urinary catheters by expediting these steps are primarily of two types ${ }^{1}$ : 'reminders' which function to simply remind that a urinary catheter is in place and should be removed if unnecessary ${ }^{2-5}$ and 'stop orders' which prompt nurses or physicians to remove catheters based on criteria, such as 24$48 \mathrm{~h}$ after surgery ${ }^{6} 7$ or when the patient no longer meets other clinical criteria. ${ }^{8-12}$ These interventions can be implemented using a range of technology, as complex as a computer-generated reminder or stop order $^{8}$ 13-15 initiated with each catheter order, or as simple as printed postprocedure order sets ${ }^{6}$ with stop orders, sticker reminders on charts ${ }^{2}{ }^{4}$ or catheter bags, ${ }^{16}$ a mandatory daily verbal ${ }^{3}$ reminder from nurses to physicians of catheterised patients, or as a reminder on a checklist ${ }^{617}$ 18 used daily on rounds. These interventions can be directed at either physicians ${ }^{4}$ 1314 or nurses, with nurse-directed interventions ranging from requiring nurses to remind physicians to order catheter removal $^{6}{ }^{17-20}$ to nurse-empowered stop orders $^{9-12} 2122$ enabling nurses to remove catheters that do not meet appropriate criteria, without requiring an additional physician order. Consequently, there are many options to consider when designing an intervention to facilitate removal of unnecessary catheters, with success demonstrated using all of these options.

The article by Janzen $e t a l^{23}$ in this issue of the journal describes an intervention aimed at expediting the process of catheter removal by increasing physician awareness through educational sessions and requiring daily review of catheter necessity, with nurses encouraged to remind physicians to remove catheters. With a significant decrease in catheterisation duration, catheter-associated urinary tract infections (CAUTIs), and even length of stay, readers may be inspired to try similar strategies for their own hospitals with high expectations. But will it work as well? Unfortunately, not all types of urinary catheter reminders (or stop orders) are equally effective. The success of reminders and stop orders in reducing urinary catheter use depends upon the environment in which they are deployed. Because both physicians and nurses have preferences (which may conflict) regarding urinary catheter use and removal, the success of interventions to reduce catheter use depends on how concerns and challenges that arise are addressed, and whether reducing catheter use is seen as a common goal for the team of physicians and nurses, or as a lowpriority recommendation approached with resistance or indifference. Thus, whether the environment promotes respectful communication and problem solving versus turf battles and accountability wars will influence the impact of interventions for reducing urinary catheter use.

Because removing catheters requires changes in longstanding caregiver habits, perhaps the most important 'environment' influencing the success of a reminder intervention is the pre-existing behaviour and communication patterns of the physicians and nurses. In other words, how much change is required in the routine workflow of the physicians and nurses for the intervention to succeed? For example, Janzen et $a l^{23}$ describe what sounds like a simple intervention to increase physician awareness of unnecessary catheters by educational sessions, posters, and encouraging nurses to remind physicians to remove the catheters-all of which could be expected 
to be low-cost, low-resource-requiring interventions that could be implemented anywhere. Yet, perhaps the most important aspect of this intervention's success is that the daily review of catheter necessity occurred as an addition to routine daily face-to-face communication between the nurse and physician at the bedside for general medicine ward patients. In many hospitals, such routine daily bedside nurse-physician conversations and use of a checklist to review complication prevention are more typical of intensive care unit (ICU) teams, ${ }^{6} 111718$ and less commonly seen on general wards where communication between nurse and physician may occur primarily through means of chart orders and pages.

Routine face-to-face communication at the bedside between nurses and physicians has the potential to facilitate catheter removal in several aspects. Because the conversation occurs at the patient's bedside, it is less of a challenge to recognise the patient has a urinary catheter in place by checking under the sheet, which addresses the first step in getting catheters out. It may be easier to re-assess the indication for the catheter in the patient's presence and discuss feasibility using catheter alternatives, which can vary by patient characteristics such as genitourinary anatomy, mental status, and whether the patient can be safely turned routinely. Face-to-face communication increases accountability for the catheter, and has the potential to address nurse reluctance to remove catheters when related to lack of confidence in assessing catheter need. Therefore, although Janzen et $a l^{23}$ describe the reminder intervention as a simple daily review of catheter use and recommended reminder by nurses to physicians, the success of this intervention may reflect its implementation in the context of an established routine of daily bedside conversation, rather than expecting physicians or nurses to review independently, or nurses to contact physicians outside of existing routine interactions to remind them about catheter removal.

Janzen $e t a l^{23}$ deserve to be congratulated because they focused on one of the most important tasks in preventing CAUTIs (removal of unnecessary catheters), ${ }^{24}$ but also because the design of their intervention was appropriate with strong potential for success in the context of their environment (eg, established face-to-face bedside communication). Yet, readers are cautioned that implementation of catheter reminders can be more challenging in other environments. Considering methods to facilitate behaviour change, communication and accountability in addition to implementing the specific reminder intervention is critical for success. For example, implementing a unit policy that requires daily review of catheter necessity or an electronic reminder to physicians or nurses to consider removing a catheter may be less successful than in Janzen et $a l^{23}$ because of less structure in expectations of when this review would occur and how physicians and nurses should communicate about catheter removal or uncertainty about catheter alternatives, supporting the default of the catheter remaining in place.

Adding a reminder intervention to a pre-existing bedside communication between physicians and nurses (similar to adding an item to a daily checklist used in ICU rounds) may be the ideal circumstance to employ a reminder. In this setting, the reminder is targeted to the correct clinicians making decisions for the patient that day, the topic is reviewed when other clinical assessments and decisions are made impacting the necessity of the catheter, and it is not as easy to ignore when incorporated into the routine for all patients. Interventions that serve to remind about catheter presence or prompt catheter removal have the potential to succeed using either high ${ }^{813-15}$ or low ${ }^{2-4} 7916$ technology strategies, if implemented in an environment that supports the clinical review and communication required to facilitate removing catheters.

In environments without routine face-to-face bedside communication between nurses and physicians, catheter stop orders that require action by physicians or nurses may facilitate the four steps required to remove catheters better than reminders alone. Nurse-empowered stop orders ${ }^{8-12} 2122$ that allow nurses to remove catheters by criteria without requiring a physician order have potential to bypass the first three steps required to remove the catheter. However, the success of nurse-empowered stop orders is highly dependent upon nurse comfort ${ }^{22}$ with the autonomy to assess catheter need and also the resources and leadership provided to reduce the temptations to use indwelling catheters. Nurse-to-patient ratios may influence the temptation to use catheters to manage incontinence because noncatheter alternatives may require more bedside tasks and time. Nursing comfort and enthusiasm for alternatives to indwelling catheters is an important contributor to the success of interventions to reduce catheter use. ${ }^{25}$ Interventions can be impeded by physician behaviour, particularly if physicians do not support nursing efforts to reduce catheter use. Therefore, another important aspect of the environment that influences the success of a catheter-reducing intervention is the specific patient safety culture, including the level of respect between nurses and physicians, along with their willingness to try new strategies and prioritisation of preventing complications among the day's tasks. Although rarely assessed or described in intervention studies, the patient safety culture in which the intervention is implemented is a key component of its success and generalisability to other settings.

The implementation of catheter reminders and stop orders is also facilitated by the use of dedicated personnel to review, remind and reinforce the recommendations to remove unnecessary urinary catheters. In some studies, ${ }^{15}{ }^{26}$ this task is performed by a specific nurse (eg, 'Foley catheter nurse') or team ${ }^{27}$ (eg, 'catheter patrol') who review whether the 
reminder or stop order is being executed as intended for each catheterised patient. In other studies, this task is facilitated less formally by a 'champion' who is often a nurse or physician chosen to motivate colleagues to make behaviour change, often by modelling how to manage patients using catheter alternatives. In Janzen $e t a l^{23}$ a research nurse is described as collecting data during daily ward visits, including receiving some data by nursing staff. It is unclear if this interaction between the research nurse and the nursing staff influenced catheter removal. Because the intervention's success could be increased by anything that improved awareness of catheters and the recommendation to remove unnecessary catheters, daily observation by a research nurse could hypothetically also improve implementation of the reminder intervention.

Implementation of interventions to reduce catheter use and CAUTI rates is also influenced by health information technology (HIT), which can facilitate data collection regarding catheter use and CAUTI rates, expediting feedback of data to teams to help motivate and sustain efforts to reduce catheter use. However, reliance on HIT to assess and feedback catheter use and CAUTI rates to teams can also be a barrier: a large amount of people and financial resources can be expended to generate and disseminate data reports from HIT, with these tasks becoming the focus of the efforts rather than tackling the challenge of changing clinical practice. These data reports can become the primary form of communication between hospital leadership or infection prevention teams and the frontline clinicians, with little communication occurring regarding how the reports are or should be used, and what specific resources the clinical teams need to facilitate practice change. The data regarding urinary catheter use and CAUTI rates are not more valuable when generated automatically by a complex HIT system compared with a daily bed census of patients with catheters collected on paper by a nurse or clerk in a hospital with less HIT resources. In fact, HIT-generated reports regarding catheter use may seem less relevant to ward teams when deluged with other HIT-generated reports competing for their attention, particularly if they do not have confidence that the data accurately capture their own patients. Also, although larger hospitals often have more sophisticated HIT systems, a request to improve the data collection or reports regarding urinary catheter use may have low priority in the long queue of requests to information technology teams overall, leading to delays in data collection and implementation dependent on HIT. In contrast, though a smaller hospital may have a HIT system with fewer bells and whistles to generate reports and electronic reminders or stop orders, these systems can sometimes be modified faster for improved data collection.

The success and sustainability of implementing interventions such as catheter reminders or stop orders is also influenced by staff turnover and staff expertise. Smaller and rural hospitals have more difficulty recruiting and maintaining physicians. ${ }^{28} \mathrm{~A}$ frequently changing panel of physicians necessitates recurring staff education regarding patient safety expectations and protocols, with more difficulty holding physicians accountable or engaging physicians as leaders. Smaller and rural hospitals also have more difficulty recruiting staff with expertise in infection prevention, ${ }^{28}$ yet they can address the expertise gap by developing collaborative relationships with academic centres and state hospital associations, which can provide resources such as educational modules and data collection training. ${ }^{28}$ Although smaller hospitals have fewer specialised staff, the role of infection preventionist can be filled by dual-role staff such as a nurse with training in infection control. Dual roles can create time conflicts, but dual-role staff members have potential for stronger influence on behaviour change as they are recognised by the bedside staff as one of them, rather than simply instituting rules without recognition of the pragmatic difficulties implementing the changes.

Although larger, higher resource hospitals may have less difficulty recruiting and maintaining staff with expertise, the sheer size of the institution and delegation of tasks can lead to more physical and social distance between the infection prevention team and the bedside care team. Large teams also have issues with assigning accountability, as individual tasks are delegated (such as data collection or staff education) but solving the overall problem of unnecessary catheter use may be assigned to no one in particular. In contrast, in a smaller hospital, it can be clearer who is taking responsibility for the problem of catheter use, and if this person is a strong advocate for the resources they need, leadership may be more responsive, and change can occur quickly. Therefore, although small hospitals often learn from large hospitals in terms of educational and clinical expertise, large hospitals should learn from small hospitals regarding how to use personal connections and creative solutions to address the thorny issues of changing bedside behaviour.

Sustaining reductions in unnecessary urinary catheter use is also challenging, but feasible. ${ }^{78} 1525$ The literature includes several studies, like Janzen et $a l,{ }^{23}$ which report outcomes for only short post-intervention time periods (weeks to $<6$ months). ${ }^{5} \begin{array}{llllll}10 & 12-14 & 21 & 29 & 30\end{array}$ Successful interventions with sustained impact have been noted to continue to collect and feedback data on catheter use, ${ }^{25}$ are nurse directed, ${ }^{8}$ and have bedside nurses who identified themselves as being responsible or sharing responsibility for reviewing catheter necessity. $^{25}$

In summary, reminders and stop orders to prompt removal of unnecessary urinary catheters can be powerful interventions, particularly when employed in an environment that facilitates the communication and 
behaviour changes required to encourage catheter removal. The impact of a catheter reminder or stop order can range from an intermittent but quickly forgotten prompt to an invaluable lever that repeatedly expedites catheter removal, by increasing awareness of unnecessary catheters to start a cascade of actions while capitalising on effective streams of communication and staff readiness to change. When reading the next study demonstrating success in reducing unnecessary catheter use, instead of asking will it work for your team, think about why it worked for their team and how your environment could be prepared to maximise the intervention's effect. Fortunately, support and expertise $^{31-33}$ are readily available for diagnosing and optimising environments to implement interventions to reduce catheter use and CAUTIs.

Competing interests JM's research is funded by the Agency for Healthcare Research and Quality (AHRQ Award 1R010HS018344-01A1 and 1K08-HS019767-01), and she is a recipient of the National Institutes of Health Clinical Loan Repayment Program. She has also received support for the development and implementation of the 'On the CUSP: Stop CAUTI' Initiative, serving as a core national faculty through a subcontract with Health Research and Educational Trust through AHRQ. She has also received honoraria for developing and delivering lectures about catheter-associated urinary tract infections from various academic medical centers, hospitals, specialty societies, and state-based hospital associations.

Provenance and peer review Not commissioned; internally peer reviewed.

\section{REFERENCES}

1 Meddings J, Rogers MA, Macy M, et al. Systematic review and meta-analysis: reminder systems to reduce catheter-associated urinary tract infections and urinary catheter use in hospitalized patients. Clin Infect Dis 2010;51:550-60.

2 Saint S, Kaufman SR, Thompson M, et al. A reminder reduces urinary catheterization in hospitalized patients. Jt Comm J Qual Patient Saf 2005;31:455-62.

3 Apisarnthanarak A, Thongphubeth $\mathrm{K}$, Sirinvaravong S, et al. Effectiveness of multifaceted hospitalwide quality improvement programs featuring an intervention to remove unnecessary urinary catheters at a tertiary care center in Thailand. Infect Control Hosp Epidemiol 2007;28:791-8.

4 Bruminhent J, Keegan M, Lakhani A, et al. Effectiveness of a simple intervention for prevention of catheter-associated urinary tract infections in a community teaching hospital. Am J Infect Control 2010;38:689-93.

5 Crouzet J, Bertrand X, Venier AG, et al. Control of the duration of urinary catheterization: impact on catheter-associated urinary tract infection. J Hosp Infect 2007;67:253-7.

6 Fuchs MA, Sexton DJ, Thornlow DK, et al. Evaluation of an evidence-based, nurse-driven checklist to prevent hospital-acquired catheter-associated urinary tract infections in intensive care units. J Nurs Care Qual 2011;26:101-9.

7 Stephan F, Sax H, Wachsmuth M, et al. Reduction of urinary tract infection and antibiotic use after surgery: a controlled, prospective, before-after intervention study. Clin Infect Dis 2006;42:1544-51.

8 Topal J, Conklin S, Camp K, et al. Prevention of nosocomial catheter-associated urinary tract infections through computerized feedback to physicians and a nurse-directed protocol. Am J Med Qual 2005;20:121-6.

9 Loeb M, Hunt D, O’Halloran K, et al. Stop orders to reduce inappropriate urinary catheterization in hospitalized patients: a randomized controlled trial. J Gen Intern Med 2008;23:816-20.

10 Adams D, Bucior H, Day G, et al. HOUDINI: make that urinary catheter disappear-nurse-led protocol. J Infect Prev 2012;13:44-6.

11 Dumigan DG, Kohan CA, Reed CR, et al. Utilizing national nosocomial infection surveillance system data to improve urinary tract infection rates in three intensive-care units. Clin Perform Qual Health Care 1998;6:172-8.

12 Gotelli JM, Merryman P, Carr C, et al. A quality improvement project to reduce the complications associated with indwelling urinary catheters. Urologic Nursing 2008;28:465-7.

13 Cornia PB, Amory JK, Fraser S, et al. Computer-based order entry decreases duration of indwelling urinary catheterization in hospitalized patients. Am J Med 2003;114: 404-7.

14 Andreessen L, Wilde MH, Herendeen P. Preventing catheter-associated urinary tract infections in acute care: the bundle approach. J Nurs Care Qual 2012;27:209-17.

15 Knoll BM, Wright D, Ellingson L, et al. Reduction of inappropriate urinary catheter use at a Veterans Affairs hospital through a multifaceted quality improvement program. Clin Infect Dis 2011;52:1283-90.

16 Murphy D, Francis K, Litzenberger M, et al. Reducing urinary tract infection: a nurse-initiated program. Pa Nurse 2007;62:20.

17 Jain M, Miller L, Belt D, et al. Decline in ICU adverse events, nosocomial infections and cost through a quality improvement initiative focusing on teamwork and culture change. Qual Saf Health Care 2006;15:235-9.

18 Reilly L, Sullivan P, Ninni S, et al. Reducing Foley catheter device days in an intensive care unit: using the evidence to change practice. AACN Adv Crit Care 2006;17:272-83.

19 Elpern EH, Killeen K, Ketchem A, et al. Reducing use of indwelling urinary catheters and associated urinary tract infections. Am J Crit Care 2009;18:535-41; quiz 542.

20 Huang WC, Wann SR, Lin SL, et al. Catheter-associated urinary tract infections in intensive care units can be reduced by prompting physicians to remove unnecessary catheters. Infect Control Hosp Epidemiol 2004;25:974-8.

21 Voss AB. Incidence and duration of urinary catheters in hospitalized older adults: before and after implementing a geriatric protocol. J Gerontol Nurs 2009;35:35-41.

22 Wenger JE. Cultivating quality: reducing rates of catheter-associated urinary tract infection. Am J Nurs 2010;110:40-5.

23 Janzen J, Buurman B, Spanjaard L, et al. Reduction of unnecessary use of indwelling urinary catheters. BMJ Qual Saf 2013;22:984-8.

24 Meddings J, Saint S. Disrupting the life cycle of the urinary catheter. Clin Infect Dis 2011;52:1291-3.

25 Fakih MG, Rey JE, Pena ME, et al. Sustained reductions in urinary catheter use over 5 years: bedside nurses view themselves responsible for evaluation of catheter necessity. Am J Infect Control 2013;41:236-9.

26 van den Broek PJ, Wille JC, van Benthem BHB, et al. Urethral catheters: can we reduce use? BMC Urol 2011;11:10.

27 Titsworth WL, Hester J, Correia T, et al. Reduction of catheter-associated urinary tract infections among patients in a 
neurological intensive care unit: a single institution's success. J Neurosurg 2012;116:911-20.

28 Harrod M, Manojilovich M, Kowalski C, et al. Unique factors rural VA hospitals face in implementing healthcare-associated infection prevention initiatives [Issue Brief]. Veterans Rural Health Resource Center-Central Region. Washington, DC: VHA Office of Rural Health. 2013. http://www.ruralhealth.va. gov/publications.asp (accessed 15 Jun 2013).

29 Robinson S, Allen L, Barnes MR, et al. Development of an evidence-based protocol for reduction of indwelling urinary catheter usage. Medsurg Nurs 2007;16:157-61.

30 Weitzel T. To cath or not to cath? Nursing 2008;38:20-1.
31 Saint S, Olmsted RN, Fakih MG, et al. Translating health care-associated urinary tract infection prevention research into practice via the bladder bundle. Jt Comm J Qual Patient Saf 2009;35:449-55.

32 Catheterout.org Toolkit. http://www.catheterout.org (accessed 22 Jun 2013).

33 National Implementation of the Comprehensive Unit-based Safety Program (CUSP) to Eliminate Health Care-Associated Infections. On the CUSP: stop CAUTI toolkit and resources. http://www.onthecuspstophai.org/ on-the-cuspstop-cauti/toolkits-and-resources (accessed 21 Jun 2013). 